\title{
Mechanism and biological significance of the overexpression of IFITM3 in gastric cancer
}

\author{
JUN HU, SHUWEI WANG, YAN ZHAO, QINHAO GUO, DONGSHENG ZHANG, \\ JIEJING CHEN, JUAN LI, QIANG FEI and YUEMING SUN
}

\begin{abstract}
Department of Colorectal Surgery, The First Affiliated Hospital of Nanjing Medical University, Nanjing, Jiangsu 210029, P.R. China
\end{abstract}

Received July 2, 2014; Accepted August 28, 2014

DOI: $10.3892 / o r .2014 .3522$

\begin{abstract}
Interferon-induced transmembrane protein 3 (IFITM3) has been recently identified as a potential molecular marker. IFITM3 has been reported to be upregulated in various human diseases, including colon and breast cancer, astrocytoma, as well as ulcerative colitis. However, the clinical significance and underlying mechanisms of IFITM3 dysregulated expression in gastric cancer (GC) remain to be determined. The present study aimed to evaluate the expression of IFITM3 in human gastric tumor cells and specimens and investigate the effects of IFITM3 knockdown in the regulation of GC growth and its potential mechanism. IFITM3 expression was significantly overexpressed in the GC cell lines and GC tissues compared with corresponding normal controls by RT-qPCR, western blot analysis and immunohistochemistry, and this overexpression was correlated with tumor differentiation, lymph node and distant metastasis, and advanced tumor node metastasis stages. Furthermore, knockdown of IFITM3 expression suppressed tumor cell migration, invasion and proliferation significantly in vitro, arrested tumor cells at the G0/G1 phase and reduced the cell numbers in the $S$ phase of the cell cycle. We preliminarily confirmed that IFITM3 can be mediated by the activities of $\mathrm{Wnt} / \beta$-catenin signaling. Further investigation revealed that silencing of IFITM3 effectively reversed the epithelial-to-mesenchymal transition (EMT) phenotype and reduced the activities of MMP-2 and MMP-9 expression. Taken together, these data suggested that IFITM3 is a potential therapeutic target for GC.
\end{abstract}

Correspondence to: Dr Yueming Sun, Department of Colorectal Surgery, The First Affiliated Hospital of Nanjing Medical University, 300 Guangzhou Road, Nanjing, Jiangsu 210029, P.R. China E-mail: sunyueming1964@126.com

Abbreviations: AJCC, American Joint Committee on Cancer; EMT, epithelial-to-mesenchymal transition; mRNA, messenger RNA; MMPs, matrix metalloproteinases

Key words: gastric cancer, matrix metalloproteinase, epithelial-tomesenchymal transition, interferon-induced transmembrane protein 3 , Wnt/ $\beta$-catenin signaling pathway

\section{Introduction}

Gastric carcinoma is the second leading cause of cancer-related mortality and the fourth most common type of cancer worldwide $(1,2)$. There are over 750,000 new cases diagnosed annually worldwide (3), and the overall 5-year survival rate remains low (4). Although progress has been made, the pathophysiological mechanisms and reliable molecular biomarkers of gastric cancer (GC) remain to be clarified. Therefore, relevant molecular and genetic studies to establish effective control of the initiation and progression of GC are imperative.

Interferon-induced transmembrane protein 3 (IFITM3), also known as $1-8 \mathrm{U}$, is a member of the IFN-inducible transmembrane protein family. The IFITM3 gene was initially isolated from a genetic screen to identify the genes involved in the acquisition of germ-cell competence (5). Previously, it was shown that IFITM3 belongs to a family of murine genes (6), which consists of short, two transmembrane domain proteins (5-18 kDa) with a high core sequence similarity but divergent $\mathrm{N}$ - and C-termini. The human homologues including IFITM1, IFITM2 and IFITM3 are clustered on chromosome 11 within an 18-kb genomic sequence (7-9). Moreover, the IFITMs play an important role in different cell processes, including cell adhesion, immune-cell regulation, germ-cell homing and maturation, and bone mineralization (10-14).

The function of the IFITMs in tumorigenesis was previously confirmed. For example, the expression of IFITM1 and IFITM3 was significantly upregulated in astrocytoma cells compared to normal astrocytes in mice $(11,15,16)$ and IFITM1 overexpression was promoted, with IFITM1 knockdown suppressing the invasion of HNSCC cells (17). Furthermore, IFITM2 as an independent pro-apoptotic p53 gene has played a vital role in regulating the tumor cell death pathway (18). However, the concrete function and potential mechanisms of IFITM3 in GC pathogenesis are unclear. In the present study, we aimed to determine the IFITM3 expression level and its regulation mechanism in GC.

\section{Materials and methods}

Human tissue specimens. Forty-eight specimens of GC tissues and adjacent non-cancer tissues were surgically obtained between June 2012 and December 2013 at the First Affiliated 
Hospital of Nanjing Medical University (Jiangsu, China). The corresponding normal gastric tissue samples were extracted $>5 \mathrm{~cm}$ from the edge of the tumor, while no evident organization of the tumor cells was detectable. Resected tissue samples were immediately frozen in liquid nitrogen, and stored at $-80^{\circ} \mathrm{C}$ until the extraction of total RNA. TNM disease stage was classified according to The American Joint Committee on Cancer (AJCC), 7th edition. In the present study, patients that had undergone any preoperative treatments were not included.

Cell lines and cell culture. The GES-1 human gastric epithelial mucosa cell line, and SGC7901, MKN28, MKN45, AGS and BGC823 GC cell lines were obtained in our general laboratory. The cells were cultured in RPMI-1640 containing 10\% fetal bovine serum (FBS), penicillin $(100 \mathrm{U} / \mathrm{ml})$ and streptomycin $(100 \mu \mathrm{g} / \mathrm{ml})$ under cell culture conditions $\left(5 \% \mathrm{CO}_{2}, 95 \%\right.$ relative humidity and $37^{\circ} \mathrm{C}$ ).

Quantitative real-time PCR (RT-qPCR). Total RNA was isolated from cell cultures or tissues using TRIzol reagent (Invitrogen, Carlsbad, CA, USA). First-strand complementary DNA (cDNA) was reverse-transcribed using the PrimeScript RT kit (Takara, Dalian, China). RT-qPCR was performed with FastStart Universal SYBR-Green Master (Rox) (Roche Diagnostics, Indianapolis, IN, USA) with an ABI 7500 (Applied Biosystems, Life Technologies Corporation, Carlsbad, CA, USA). The specific oligonucleotide primers are listed in Table I. PCR was performed using the following cycles: $95^{\circ} \mathrm{C}$ for $30 \mathrm{sec}, 40 \mathrm{cycles}$ of $95^{\circ} \mathrm{C}$ for $5 \mathrm{sec}, 60^{\circ} \mathrm{C}$ for $30 \mathrm{sec}$; and the dissociation stage: $95^{\circ} \mathrm{C}$ for $15 \mathrm{sec}, 60^{\circ} \mathrm{C}$ for $1 \mathrm{~min}$ and $95^{\circ} \mathrm{C}$ for $15 \mathrm{sec}$. Each sample was analyzed in triplicate.

Lentivirus packaging and stable transfection cell line generation. To investigate the overexpression of IFITM3, we modified LV-IFITM3 vector lentiviral constructs (Shanghai GenePharma Co., Ltd., Shanghai, China) to knock down IFITM3 in GC cells. The empty lentiviral (LV-NC) with enhanced green fluorescent protein (EGFP) served as a negative control. The constructed vectors were verified by DNA sequencing. The GC cells were infected with LV-IFITM3 and LV-NC. The supernatant was removed after $24 \mathrm{~h}$ and replaced with fresh culture medium. The cells were selected with $2 \mu \mathrm{g} / \mathrm{ml}$ puromycin for 2 weeks.

Wound-healing assay. Cells $\left(5 \times 10^{5}\right)$ were seeded in 6-well plate and incubated for $24 \mathrm{~h}$. When the cells were grown to $90-100 \%$ confluency, a linear wound was generated by scratching the adherent cells using a sterile plastic pipette tip. The medium was displaced with serum-free medium. Immediately and $24 \mathrm{~h}$ after incubation at $37^{\circ} \mathrm{C}$, migrating cells at the wound front were photographed using an inverted microscope. Each experiment was performed at least three times in triplicate.

Transwell migration and invasion assay. In vitro invasion was determined using 24-well Transwell chamber. Cells $\left(2 \times 10^{4}\right)$ were suspended in $100 \mu \mathrm{l}$ of serum-free medium and placed in the upper compartment of a Transwell chamber with 8-mm membrane filter inserts coated with Matrigel (BD Biosciences). Subsequently, the medium with $10 \%$ FBS was added to the lower chamber as a chemoattractant. After incubation for $24 \mathrm{~h}$,
Table I. Oligonucleotide primer sequences used in the RT-qPCR.

\begin{tabular}{|c|c|c|}
\hline $\begin{array}{l}\text { RT-qPCR } \\
\text { primer }\end{array}$ & Sequence & $\begin{array}{l}\text { Product } \\
\text { length } \\
\text { (bp) }\end{array}$ \\
\hline
\end{tabular}

IFITM3 F: 5'-CGAAACTACTGGGGAAAGGGA-3'

R: 5'-ATTCATGGTGTCCAGCGAAGA-3'

$\beta$-catenin F: 5'-AAAGCGGCTGTTAGTCACTGG-3'

R: 5'-CGAGTCATTGCATACTGTCCAT-3'

E-cadherin F: 5'-GACCGAGAGAGTTTCCCTACG-3'

R: 5'-TCAGGCACCTGACCCTTGTA-3'

Vimentin F: 5'-ATGACCGCTTCGCCAACTAC-3'

178 R: 5'-CGGGCTTTGTCGTTGGTTAG-3'

MMP-2 F: 5'-TACAGGATCATTGGCTACACACC-3' R: 5'-GGTCACATCGCTCCAGACT-3'

MMP-9 F: 5'-TGTACCGCTATGGTTACACTCG-3' 97 R: 5'-RGGCAGGGACAGTTGCTTCT-3'

IFITM3, interferon-induced transmembrane protein 3.

the cells on the upper surface of the membrane filter were removed, and the cells that had penetrated to the lower surface of the membrane were fixed and stained with crystal violet. Five visual fields of each insert were randomly selected and counted under a light microscope. Migration assays were performed using a Transwell compartment, and with the exception of Matrigel, all other steps were identical. For each experimental condition, the assay was performed at least in triplicate.

Cell Counting Kit-8 (CCK-8) assay. Cell proliferation was measured by the CCK-8 (Beyotime Institute of Biotechnology, Shanghai, China) according to the manufacturer's instructions. Cells were seeded in a 96 -well plate at $5 \times 10^{3}$ cells/well with $100 \mu \mathrm{l}$ of complete culture medium. After adhesion for $24 \mathrm{~h}$, $10 \mu \mathrm{l} \mathrm{CCK}-8$ was added to each well and incubated at $37^{\circ} \mathrm{C}$ for $2 \mathrm{~h}$. The absorbance was measured at $450 \mathrm{~nm}$ using a microplate reader. Experiments were performed in triplicate and repeated three times.

Cell cycle assay. Cells ( $3 \times 10^{5}$ cells/well) were seeded in 6-well plates and cultured for $24 \mathrm{~h}$. After adhesion, the cells were harvested and centrifuged at 1,500 rpm for $5 \mathrm{~min}$. Pellets were washed with cold phosphate-buffered saline (PBS) and fixed with cold $70 \%$ ethanol at $4^{\circ} \mathrm{C}$ for $30 \mathrm{~min}$ and stored at $-20^{\circ} \mathrm{C}$ overnight. The following day, the cells were centrifuged at $1,500 \mathrm{rpm}$ for $5 \mathrm{~min}$ to remove ethanol and washed with PBS. The supernatant was discarded and propidium iodide (PI) containing RNase $\mathrm{A}$ was added at $4^{\circ} \mathrm{C}$ for $30 \mathrm{~min}$ in the dark. Cell suspension was filtered through a mesh filter and analyzed by flow cytometry.

Western blot analysis. Cultured cells were lysed using RIPA buffer (Beyotime Institute of Biotechnology), and equivalent amounts of protein were subjected to SDS-PAGE separation and subsequently diverted to polyvinylidene fluoride (PVDF) membranes (Millipore, Bedford, MA, USA). The membranes 
A

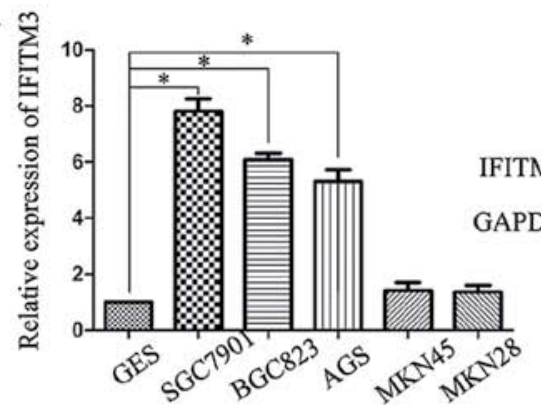

$\mathrm{C}$

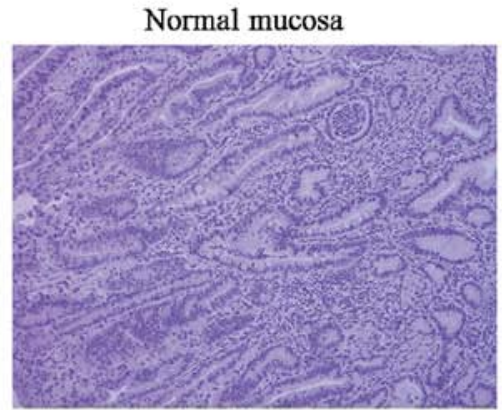

FITM3

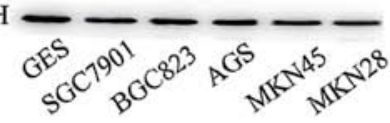

B
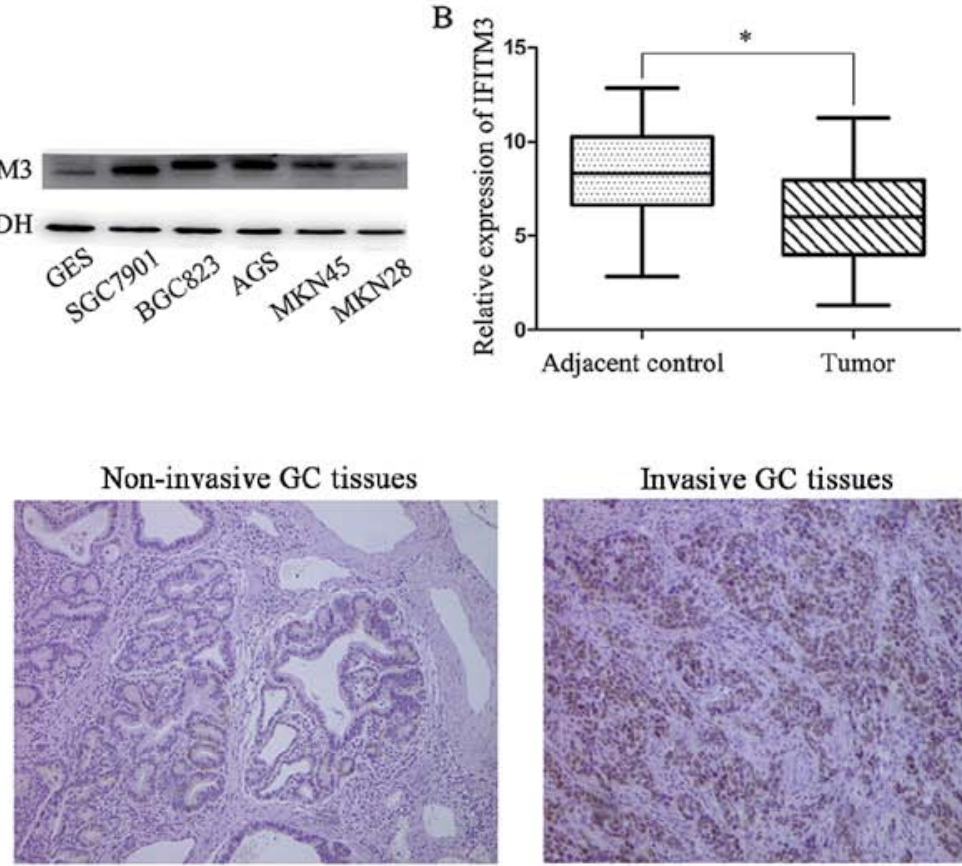

Invasive GC tissues

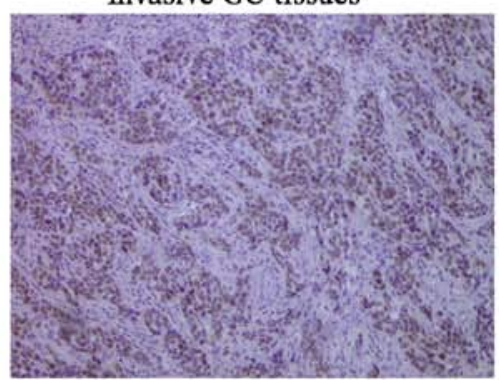

Figure 1. Expression of IFITM3 in GC tissues and cell lines. (A) IFITM3 expression was evaluated by RT-qPCR and western blot analysis in one immortalized gastric cell line (GES-1) and five GC cell lines (GES, SGC7901, BGC823, AGS, MKN45 and MKN28). $\beta$-actin and GAPDH were used as internal control, respectively. (B) IFITM3 expression levels were assessed in tumor tissues and adjacent normal tissues ( $\mathrm{n}=48$ ). The $\Delta \mathrm{CT}$ value was determined by subtracting the $\beta$-actin CT value from the IFITM3 CT value. Smaller $\Delta$ CT value indicates higher expression. (C) Immunohistochemical results of IFITM3 expression were detected in normal mucosa, non-invasive GC and invasive GC tissues. Magnification, x100. IFITM3, interferon-induced transmembrane protein 3; GC, gastric cancer.

were blocked with 5\% non-fat milk in Tris-buffered saline solution containing $0.05 \%$ Tween-20 and then incubated with antibody-specific IFITM3 (1:1,000; ab109429, Abcam), E-cadherin, vimentin, $\beta$-catenin, MMP-2, MMP-9 $(1: 1,000)$, GAPDH $(1: 10,000)$ (both from Cell Signaling Technology). The membranes were washed three times with TBST, and incubated with horseradish peroxidase (HRP)-conjugated secondary antibody (1:1,000; Beijing Biosynthesis Biotechnology) at $37^{\circ} \mathrm{C}$ for $2 \mathrm{~h}$. Bound proteins were visualized using ECL (Pierce) after three TBST washes and detected using a Bio-Imaging System. GAPDH was used as an internal loading control.

Immunohistochemistry (IHC). For immunohistochemistry, the tissue samples were fixed in $10 \%$ formalin solution and embedded in paraffin. Previous sections of $3 \mathrm{~mm}$ were prepared, then deparaffinized in xylene, and rehydrated through a graded series of ethanol. Endogenous peroxidase was blocked with $0.3 \%$ hydrogen peroxide in methanol for $20 \mathrm{~min}$ at room temperature. For pretreatment, antigen retrieval sections were performed in citrate buffer $(\mathrm{pH} 6.0)$ using a microwave for $10 \mathrm{~min}$. Overnight incubation at $4^{\circ} \mathrm{C}$ was carried out for the binding of primary antibodies (IFITM3, 1:400; ab109429, Abcam). The secondary anti-mouse antibody was subsequently applied to the sections for $30 \mathrm{~min}$ at room temperature. After rinsing with TBST, the slides were treated with diaminobenzidine solution and counterstained with hematoxylin. Immunostaining intensity ( 0 , no staining; 1, weak staining; 2 , moderate staining; and 3, strong staining) as well as the percentage of cells stained ( 0 , no cells; $1,<10 \%$ of cells; $2,11-50 \%$ of cells; $3,51-80 \%$ of cells; and $4,>80 \%$ of cells) were evaluated.
Statistical analysis. The Statistical Program for Social Sciences (SPSS) 20.0 software (IBM, SPSS, Inc., Chicago, IL, USA) was used for the statistical analysis. The data were expressed as means \pm standard deviation (SD). Differences were analyzed with the unpaired Student's t-test and analysis of variance (ANOVA). A two-tailed value of $\mathrm{P}<0.05$ was considered to indicate a statistically significant result.

\section{Results}

IFITM3 overexpression is identified in GC tissues and cell lines. To examine the role of IFITM3 in GC progression, the mRNA and protein expression of IFITM3 in the AGS, SGC7901, BGC823, MKN45 and MKN28 human GC cell lines and the GES-1 human gastric epithelial mucosa cell line were analyzed by RT-qPCR and western blot analysis, respectively. As shown in Fig. 1A, the expression levels of IFITM3 mRNA and protein were significantly higher in SGC7901 and BGC823 cell lines than in normal cell lines. However, there was no significant difference for MKN28 and MKN45. Thus, SGC7901 and BGC823 were selected as the optimal experimental groups. Furthermore, we examined the expression in 48 pairs of GC tissues compared with adjacent normal tissues. IFITM3 levels were markedly upregulated in cancer tissues compared with corresponding non-cancer tissues (Fig. 1B). The potential related factors affecting IFITM3 expression were determined and it was confirmed that IFITM3 expression was significantly associated with tumor differentiation, lymph node and distant metastasis, and TNM stages. Nevertheless, there was no significant correlation between IFITM3 expression and other clinicopathologic 
Table II. Associations between IFITN3 expression $(\Delta \mathrm{CT})$ and clinicopathological characteristics of $48 \mathrm{GC}$ patients.

\begin{tabular}{|c|c|c|c|}
\hline Characteristics & $\begin{array}{c}\text { No. of } \\
\text { patients }(\%)\end{array}$ & Mean \pm SD & P-value \\
\hline Age (years) & & & 0.539 \\
\hline$\geq 60$ & 32 & $5.84 \pm 2.31$ & \\
\hline$<60$ & 16 & $6.24 \pm 1.72$ & \\
\hline Gender & & & 0.157 \\
\hline Male & 33 & $5.68 \pm 2.02$ & \\
\hline Female & 15 & $6.62 \pm 2.25$ & \\
\hline Diameter $(\mathrm{cm})$ & & & 0.685 \\
\hline$\geq 5$ (large) & 19 & $5.82 \pm 2.50$ & \\
\hline$<5$ (small) & 29 & $6.08 \pm 1.87$ & \\
\hline Location & & & 0.685 \\
\hline Cardia or body & 30 & $6.07 \pm 2.20$ & \\
\hline Antrum & 18 & $5.81 \pm 2.03$ & \\
\hline Differentiation & & & 0.009 \\
\hline Poor or not & 32 & $5.42 \pm 2.15$ & \\
\hline Well or moderate & 16 & $7.09 \pm 1.59$ & \\
\hline Lymphatic metastasis & & & 0.002 \\
\hline Present & 38 & $5.50 \pm 2.00$ & \\
\hline Absent & 10 & $7.77 \pm 1.60$ & \\
\hline Distal metastasis & & & 0.024 \\
\hline Present & 44 & $6.18 \pm 2.08$ & \\
\hline Absent & 4 & $3.71 \pm 1.00$ & \\
\hline AJCC clinical stage & & & 0.005 \\
\hline $\mathrm{I}+\mathrm{II}$ & 13 & $7.35 \pm 2.18$ & \\
\hline III+IV & 35 & $5.47 \pm 1.88$ & \\
\hline Serum CEA value & & & 0.752 \\
\hline$\geq 5(\mu \mathrm{g} / \mathrm{l})$ & 20 & $5.86 \pm 2.52$ & \\
\hline$<5(\mu \mathrm{g} / 1)$ & 28 & $6.06 \pm 1.83$ & \\
\hline
\end{tabular}

IFITM3, interferon-induced transmembrane protein 3; AJCC, The American Joint Committee on Cancer.

characteristics, such as age, gender, diameter, tumor location and CEA values (Table II). We also examined the expression of IFITM3 by immunohistochemistry and it was found that IFITM 3 was positively stained in the cytoplasm of the majority of invasive cancer specimens, whereas IFITM3 was negatively or weakly stained in the adjacent normal tissues. Furthermore, IFITM3 expression levels were increased with the pathological stages of GC (Fig. 1C).

IFITM3 knockdown is negatively regulates $G C$ cell proliferation. To demonstrate the functional role of IFITM3 in GC tumorigenesis, we infected IFITM3 shRNA into SGC7901 and BGC823 cells and examined the transfection efficiency by RT-qPCR and western blot analysis, respectively. We found that the levels of IFITM3 expression in the LV-NC were markedly higher than that of LV-IFITM3 (Fig. 2A and B). Furthermore, obvious inhibitory effects on cell proliferation were observed in IFITM3-silenced cells at 4-6 days (Fig. 2C and D). These results suggested that IFITM3 overexpression contributes to the proliferation of colon cancer cells.

Depletion of IFITM3 arrests GC cells in the G0/G1 phase. To elucidate the effect of IFITM3 downregulation on cell cycle distribution, the flow cytometry instrument analysis was performed to show the increasing proportion of G0/G1 phase in GC cells. As shown in Fig. 3A and B, there was a significant increase in the cell population at the G0/G1 phase, associated with a decrease in the cell population at the $\mathrm{G} 2 / \mathrm{M}$ and $\mathrm{S}$ phases following IFITM3 depletion ( $\mathrm{P}<0.05$; Fig. $3 \mathrm{C}$ and $\mathrm{D})$. These data suggested that depletion of IFITM3 arrests GC cells in the $\mathrm{G} 0 / \mathrm{G} 1$ phase.

IFITM3 silencing inhibits cell migration and invasion through epithelial-to-mesenchymal transition (EMT) change. To exhibit the involvement of IFITM3 in the invasion of gastric cells, wound-healing and Transwell assays were performed using SGC7901 and BGC823 cells transfected with LV-IFITM1 or LV-NC. The cell migration effect identified by the wound-healing assay showed that compared with negative control vector-transfected cells, IFITM3 knockdown significantly inhibits GC cell migration (Fig. 4A). Similar results were obtained from the Transwell assay, while transfection with LV-IFITM3 significantly suppressed the migration and invasion of SGC7901 and BGC823 cells $(\mathrm{P}<0.05$; Fig. 4B and $\mathrm{C})$. EMT, an essential cell biological program during embryonic development, contributes to cancer invasion and metastasis $(19,20)$. To confirm the role of IFITM3 in the process of GC cells, we transfected the LV-IFITM3 vector and evaluated the expression of epithelial marker E-cadherin and mesenchymal marker vimentin at mRNA and protein levels. As shown in Fig. 4D, IFITM3 knockdown cells induced round spheroids with no or few protrusions. The increased expression of E-cadherin and decreased expression of vimentin was detected in IFITM3 knockdown cells in the mRNA and protein levels (Fig. 4E and F). This suggested that IFITM3 may regulate the migration and invasion potential of the gastric cell lines by inducing EMT.

Knockdown of IFITM3 induces downregulation of MMPs. MMPs are matrix metalloproteinases that were previously shown to play a crucial role in the tumor microenvironment by enhancing cancer cell invasion, proliferation and cancer matastasis $(21,22)$. The results showed that IFITM3 is able to promote the migration and invasion of GC cancer cells. However, whether IFITM3 mediates MMP expression remains to be determined. Therefore, we examined MMP-2 and MMP-9 expression in transfected cells and negative control by qPCR and western blotting, respectively. A significant downregulation of MMP-2 and MMP-9 expression was observed in IFITM3-silencing cells, as compared with the empty lentiviral cells ( $\mathrm{P}<0.05$; Fig. 5A and $\mathrm{B})$. These results suggested that MMPs are involved in IFITM3-induced cell migration and invasion.

Involvement of the Wnt/ $\beta$-catenin pathway in the expression of IFITM3. The canonical Wnt// $\beta$-catenin pathway plays an important role in embryonic development and tumorigenesis. It has been shown that $\mathrm{Wnt} / \beta$-catenin signaling participates in the 

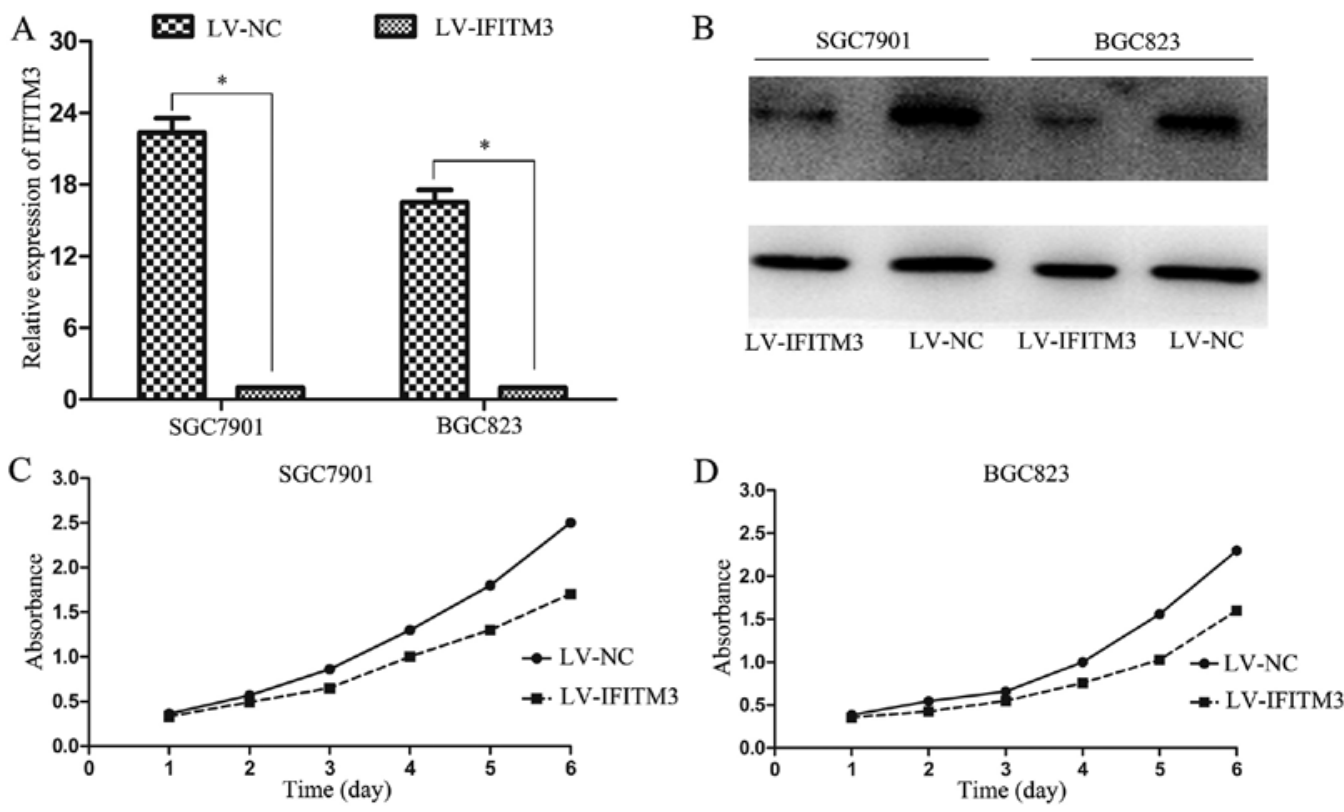

Figure 2. The effect of IFITM3 on the regulation of cell proliferation. (A) Analysis of IFITM3 expression in stable transfected GC cells using RT-qPCR. GC cells were transfected with LV-IFITM3 and LV-NC as a negative control. (B) Western blot analysis was performed to confirm the efficiency of transfected knockdown and negative control. (C and D) GC cells transfected with LV-IFITM3 or LV-NC were assayed with a CCK-8. A t-test was used to show significant differences between the two groups. " $\mathrm{P}<0.05$. IFITM3, interferon-induced transmembrane protein 3; GC, gastric cancer; CCK- 8 , Cell Counting Kit-8

$\mathbf{A}$

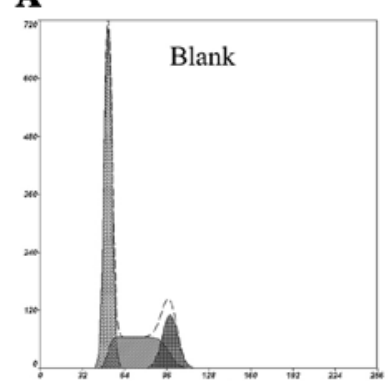

B

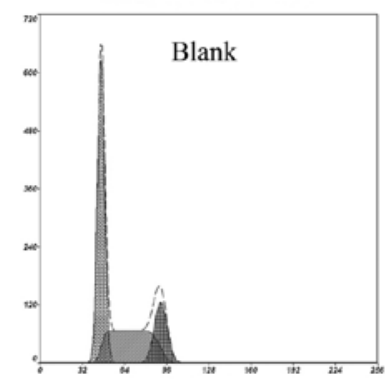

C

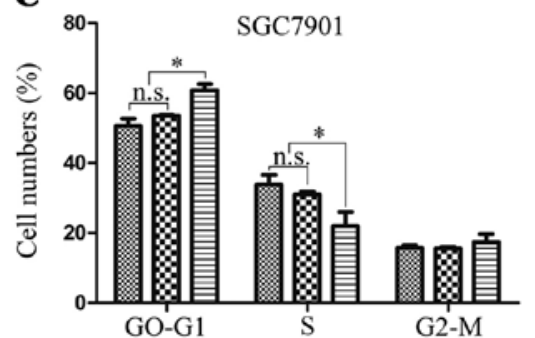

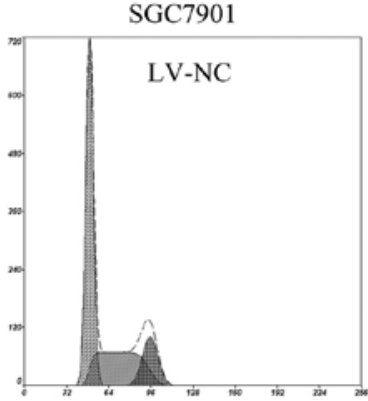
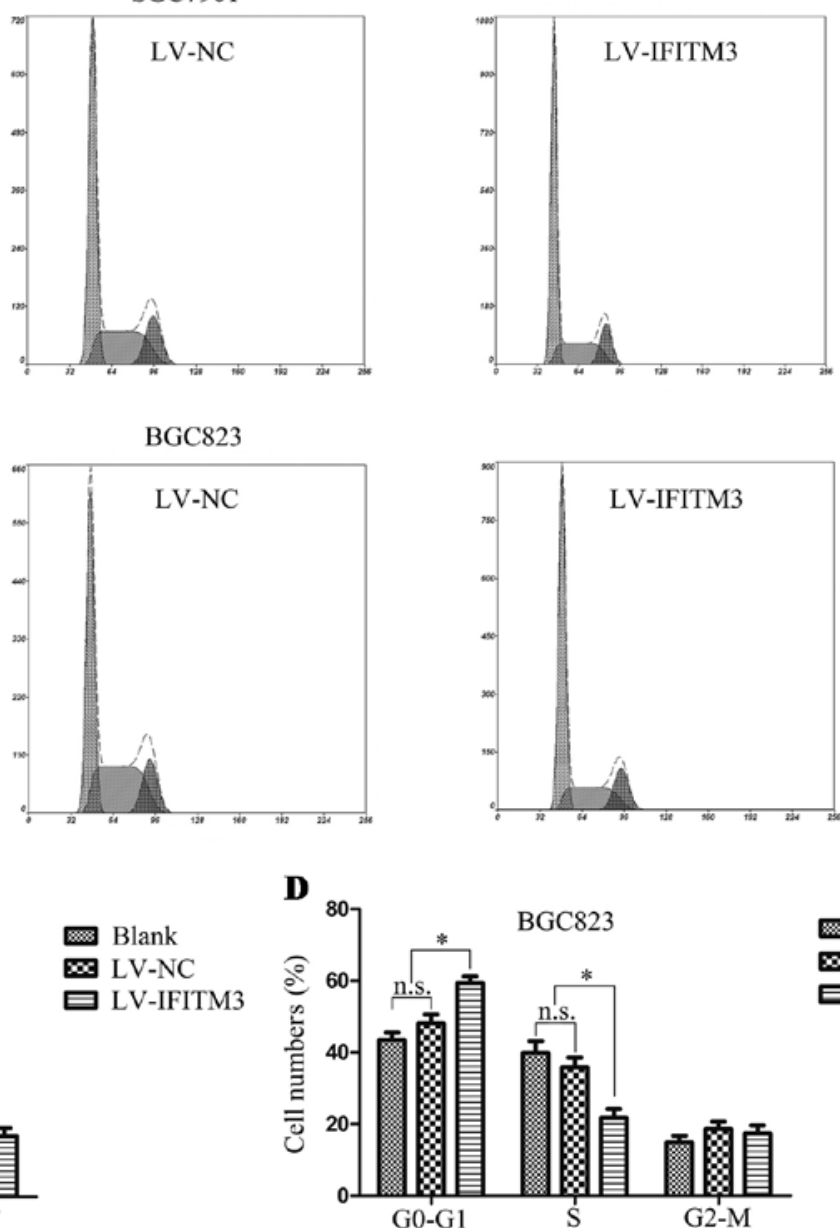

Figure 3. Effect of IFITM3 depletion on the modulation of cell cycle progression in GC cells. (A and B) Representative images of flow cytometric analysis showing IFITM3 knockdown induces G0/G1 cell cycle arrest in SGC7901 and BGC823 cells with a corresponding decrease in the S-phase cells. $(\mathrm{C}$ and $\mathrm{D})$ Summarized data of (A and B). Results are the average of three independent experiments (means $\pm \mathrm{SD})$. " $\mathrm{P}<0.05$. IFITM3, interferon-induced transmembrane protein 3; GC, gastric cancer. 
A

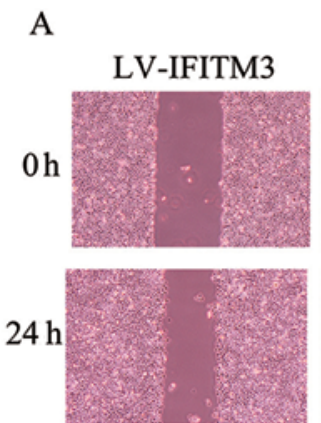

SGC7901

Blank
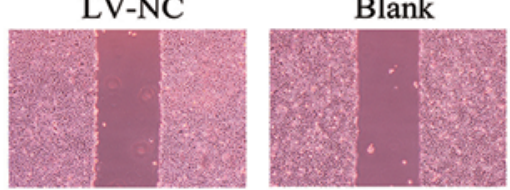

LV-IFITM3

BGC823
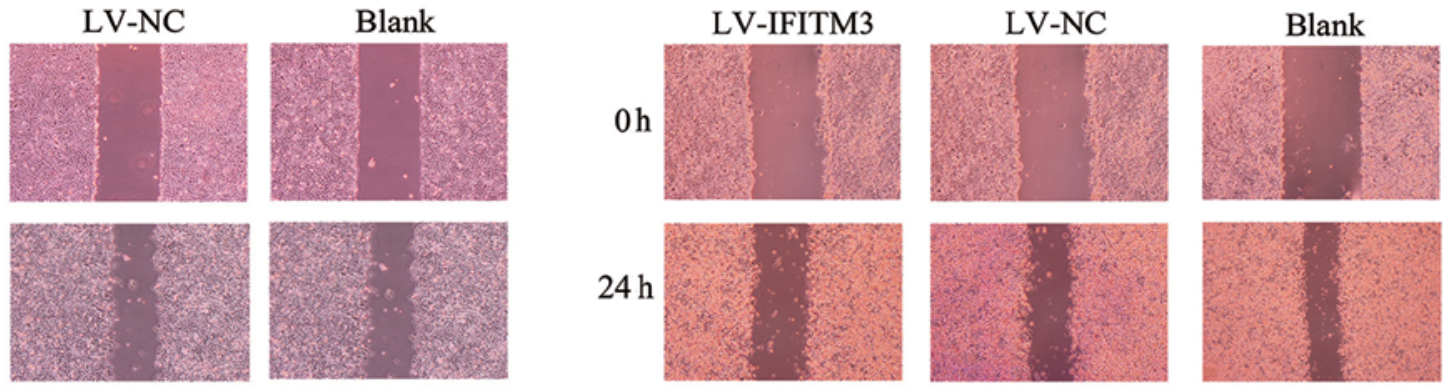

B

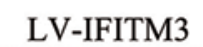

LV-NC

Blank
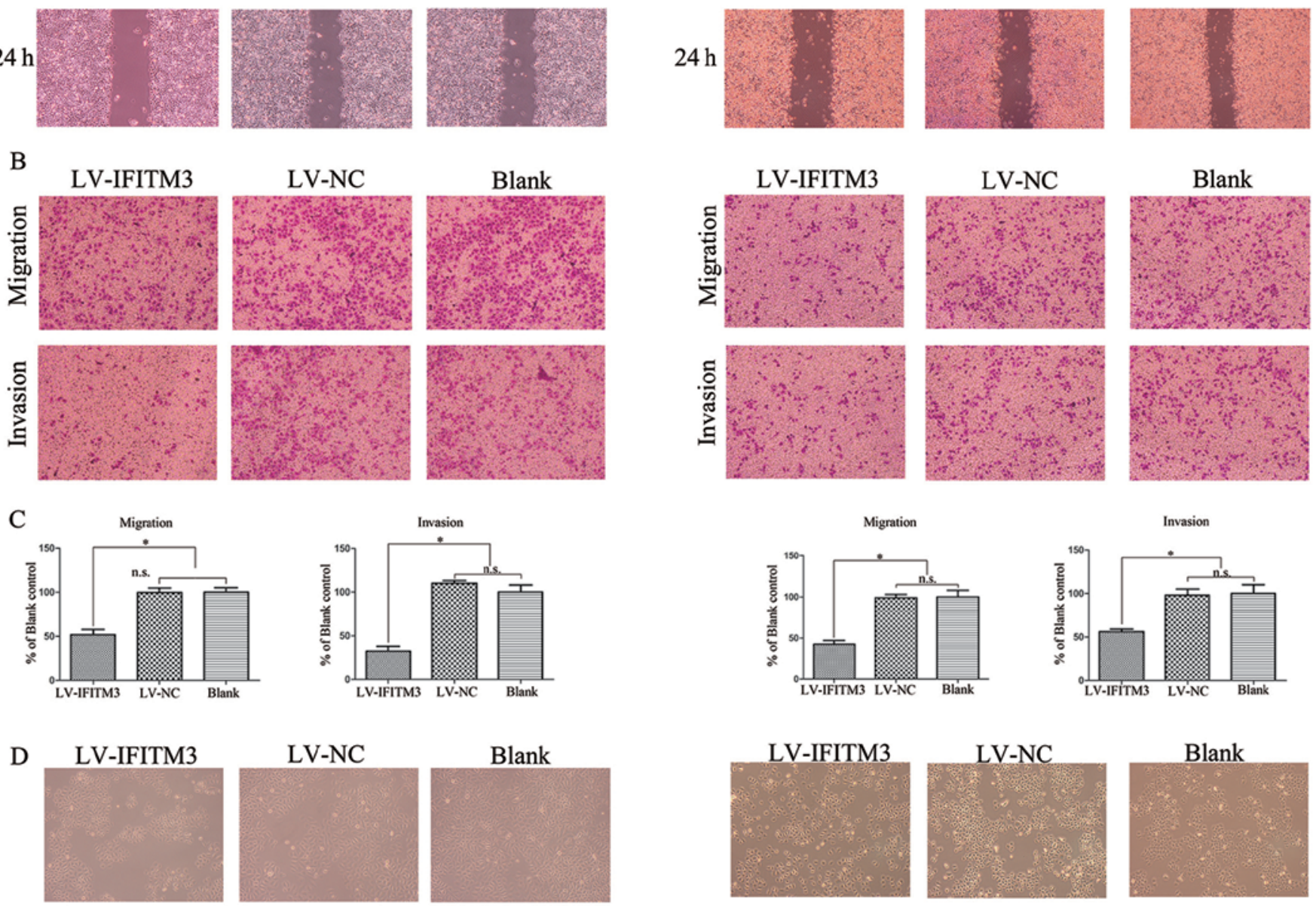

LV-NC
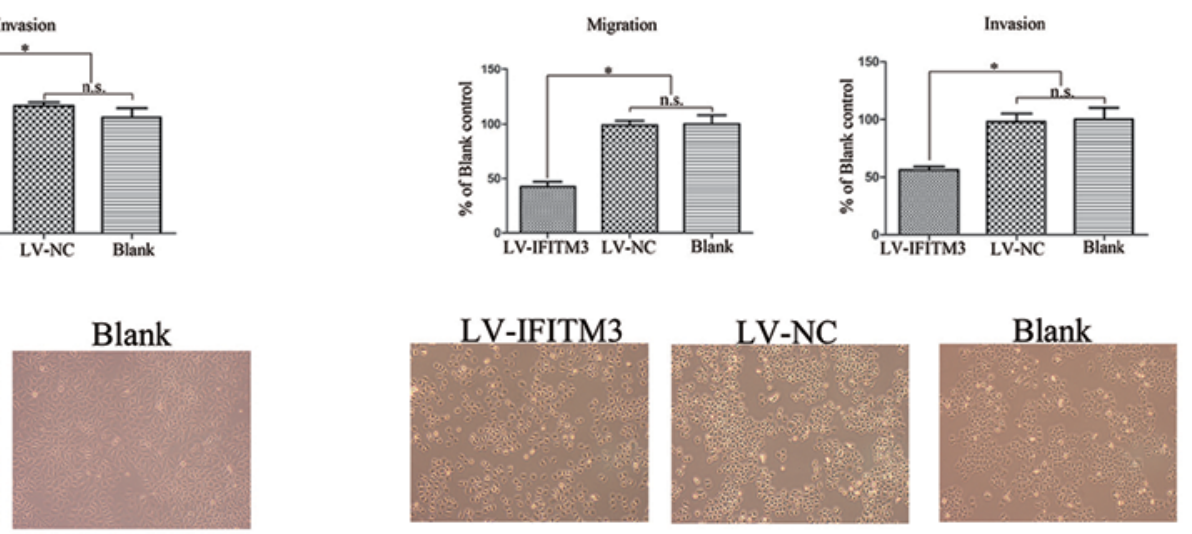

$\mathrm{E}$
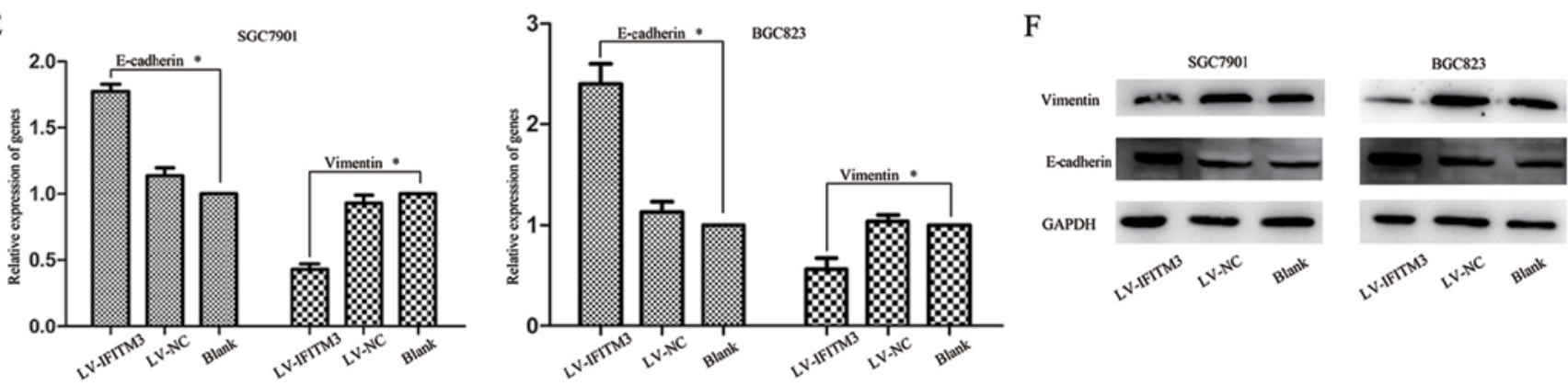

Figure 4. Regulation of GC cell migration and invasion by IFITM3. SGC7901 and BGC823 cells were transfected with IFITM3 shRNA (LV-IFITM3) or a control shRNA (LV-NC) or left untransfected (blank) were determined as described in Materials and methods. (A) Wound-healing assay with GC cells. Microscopic observations were measured at 0 and $24 \mathrm{~h}$ after the cell surface was scratched. Magnification, x40. (B) Representative microscopy images of the migration and invasion assays in GC cells. (C) Cell migration and invasion percentages. Magnification, x100. The untreated cell cultures were defined as migration and invasion percentages of $100 \%$. (D) Morphologic changes with phase-contrast photomicrographs. IFITM3-silencing-induced cobblestone-like morphology, such as the inversion of the EMT. Magnification, x100. (E) The epithelial marker E-cadherin and mesenchymal marker vimentin mRNA levels were tested in GC cell lines after IFITM3 transfection by RT-qPCR. (F) Western blot analysis was used to determine the protein levels of E-cadherin and vimentin in GC cells after HULC knockdown. "P<0.05; n.s., not significant; GC, gastric cancer; IFITM3, interferon-induced transmembrane protein 3.

regulation of IFITM genes during intestinal tumorigenesis (23). Therefore, we investigated whether the upregulation of IFITM3 in $\mathrm{GC}$ cells was induced by activation of $\mathrm{Wnt} / \beta$-catenin signaling. XAV939, a new molecular inhibitor, blocked
Wnt signaling and stable axin protein leading to $\beta$-catenin destruction (24). Concentrations of 5 and $10 \mu \mathrm{mol} / 1$, and an optimal processing time of $48 \mathrm{~h}$ were selected. GC cells were subsequently treated with various concentrations of XAV939 

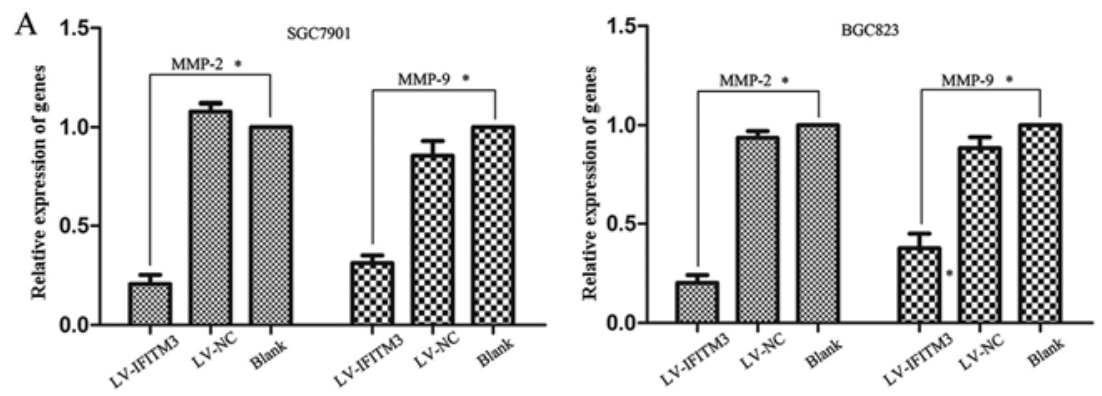

B

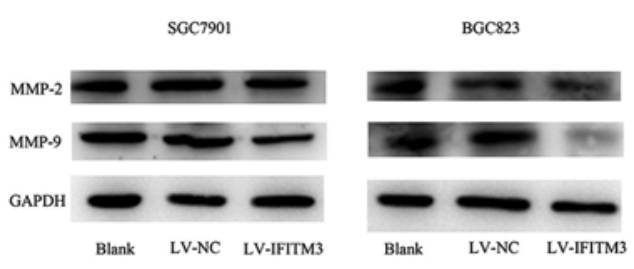

Figure 5. The expression and activity of MMPs induced by IFITM3 silencing. (A) mRNA expression for the metalloproteinases MMP-2 and MMP-9 was determined in GC cell lines after IFITM3 transfection by RT-qPCR. (B) Western blot analysis was performed to determine the protein levels of MMP-2 and MMP-9 in GC cells. " $\mathrm{P}<0.05$. IFITM3, interferon-induced transmembrane protein 3; GC, gastric cancer.
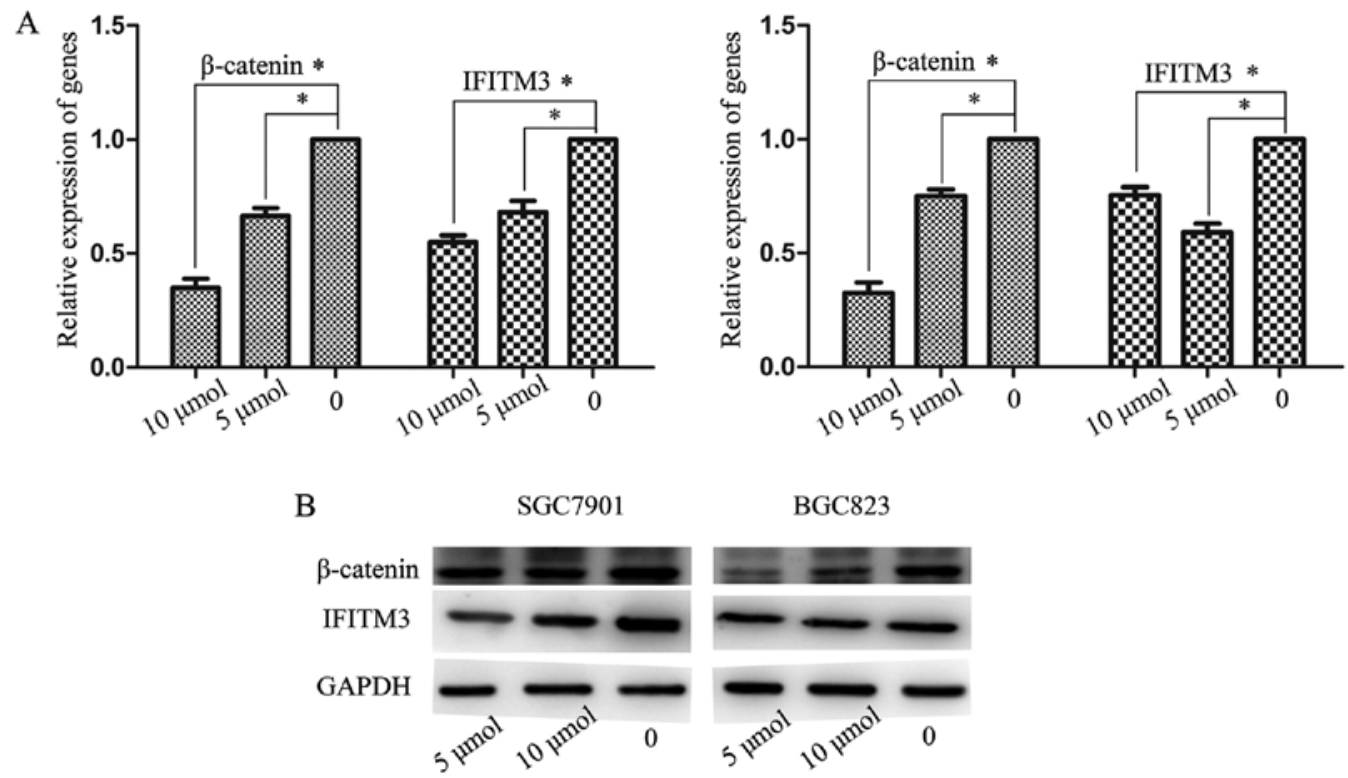

Figure 6. The involvement of the Wnt/ $\beta$-catenin pathway in IFITM3 expression. (A and B) GC cells were treated with 5 and $10 \mu \mathrm{mol} / 1 \mathrm{XAV} 939$, respectively. Levels of mRNA and protein expression of $\beta$-catenin and IFITM3 were performed after $48 \mathrm{~h}$. "P<0.05. IFITM3, interferon-induced transmembrane protein 3 ; GC, gastric cancer.

and IFITM3 expression was analyzed using RT-qPCR and western blot analysis. The results showed that the expression of $\beta$-catenin and IFITM3 maintained a high level in normal GC cells. After incubating with XAV939 with 5 and $10 \mu / \mathrm{mol}$ , IFITM3 expression markedly decreased together with the reduction of $\beta$-catenin, both of which were significantly inferior to the untreated controls $(\mathrm{P}<0.05$; Fig. $6 \mathrm{~A}$ and $\mathrm{B})$.

\section{Discussion}

IFITM3 is known to function as a cancer-promoting gene and is overexpressed in the oncogenesis of several malignancies, including glioma, colorectal and breast cancer (25-27). However, the mechanism and significance of IFITM3 is unclear. Thus, in the present study, we concretely investigated the expression of IFITM3 in GC tissues and cell lines. RT-qPCR and western blot analysis revealed that IFITM3 expression was significantly higher in SGC7901 and BGC823 cells than in normal cells at the mRNA and protein expression level. In the tissues, the level of IFITM3 expression was upregulated in GC tumor tissues, which is consistent with the adjacent normal tissues by RT-qPCR analysis. We also found that IFITM3 was significantly correlated with tumor differentiation, lymph node and distant metastasis, and TNM stages. Consistent with the abovementioned results, the results of the immunohistochemical staining indicated that IFITM3 expression was closely associated with an advanced cancer biology. IFITM3-positive staining was significantly higher in advanced GC than early GC. Alteration of IFITM3 expression offered further functional evidence that it was involved in the enhancement of aggressive biological behavior of cancer cells. There were also certain limitations in the present study. First, all of the patients were enlisted from a single institution and the number of samples was not adequate. For instance, there were only four patients at M1 stage. Additionally, since the tissues used were resected in the previous two years, the follow-up period after surgery was insufficient to investigate the relationship between IFITM3 expression and overall survival of GC patients.

IFITM3 is a double trans-membrane protein that can be upregulated by IFNs $(10,28)$. It is involved in signal transduction of antiviral, anti-inflammation, immune cell regulation 
and somitogenesis $(11,12,15,29)$. However, the precise role of IFITM3 in GC pathogenesis remains unknown. Thus, we used lentivirus-mediated RNAi to knock down IFITM3 in GC cells. shRNA targeting IFITM3 induced obvious and efficient inhibition of cell proliferation, migration and invasion. The flow cytometry data showed that the growth inhibitory effect of LV-IFITM3 was mediated by cell cycle arrest at the G0/G1 phase, and reduced the number of cells in the $\mathrm{S}$ phase. Concordant with our findings, it has previously been confirmed that IFITM1 expression promotes invasion in head and neck tumor in the early stages of disease progression (17). El-Tanani et al reported that IFITM3 reduces osteopontin mRNA expression by affecting OPN mRNA stability to modulate anchorage-independent growth, cell adhesion and invasion (30). Thus, the present study confirms that IFITM3 is important in GC tumorigenesis, suggesting IFITM3 as a potential oncogene in human GC.

Metastasis remains the leading cause of treatment failure and poor prognosis in patients with malignant tumors. It is a complex and multistage process including proteolysis, motility and migration of cells, proliferation in a new site and neoangiogenesis (31). EMT in tumor progression and metastasis may be induced by autonomous oncogenic activation or inactivation of signaling molecules with or without additional stimulation (32). During this progression, epithelial markers including E-cadherin, ZO-1 and MUC1 are downregulated and molecules such as transcription factors Snail, Slug, Twist as well as $\mathrm{N}$-cadherin and vimentin are upregulated (33). To understand whether the mechanism of IFITM3-regulated invasion and metastasis is associated with the EMT, we tested the expression of the EMT representative markers E-cadherin and vimentin treated with the control or IFITM3 knockdown. The results showed a tendency of E-cadherin to decrease and of vimentin and the morphological change (growth pattern, decreased formation of lamellipodia) to increase, suggesting that IFITM3 is probably an EMT-like phenotype. These results indicate that the alterative expression of IFITM3 had a significant impact on the process of EMT by mediating the E-cadherin and vimentin expression in GC cells. In addition, it is well known that an increased expression of MMPs is required in tumor invasion and metastasis. Among the MMPs, MMP-2 and MMP-9 are considered to be crucial enzymes in this process with degrading type IV collagen regulating various cell behaviors with relevance for cancer biology (34). Enhanced levels of MMP-2 and MMP-9 are important factors associated with metastasis and invasion in the process of GC (35). To validate the effect of IFITM3 on cell invasion and metastasis, we examined MMP-2 and MMP-9 expression in transfected cells and the negative control by qPCR and western blot analysis, respectively. Our results suggested that depletion of IFITM3 decreased the expression of MMP-2 and MMP-9. Furthermore, we confirmed that the downregulation of IFITM3 expression decreased the invasion and migration ability of GC cells. The results confirmed that changes in the expression of MMP-2 and MMP-9 were consistent with IFITM3. However, the concrete mechanisms of how IFITM3 regulates invasion and metastasis in GC cells remain to be clarified in future studies.

The Wnt/ $\beta$-catenin signaling pathway is a fundamental mechanism that regulates cell proliferation, polarity and differentiation during embryonic development. Stability of $\beta$-catenin in the cytoplasm was assessed to determine activation of the Wnt/ $\beta$-catenin signaling pathway (36). Findings of a previous study showed the expression of the IFITM family members is induced and that their transcription is dependent on the activation of $\beta$-catenin signaling in intestinal tumorigenesis (23). Thus, we hypothesize that a similar event occurs in GC. In the present study, XAV939, a specific inhibitor of $\beta$-catenin, was used to treat gastric cells at various concentrations. Our results suggest that IFITM3 expression was positively correlated with the $\beta$-catenin level, indicating that the $W n t / \beta$-catenin signaling pathway may play an important role in IFITM3 expression in GC cells and activated $\beta$-catenin signaling including IFITM3 may be involved in gastric carcinoma. Nevertheless, the molecular mechanism of IFITM3 overexpression underlying the regulating process of the Wnt/ $\beta$-catenin signaling pathway in GC remains to be elucidated. However, more studies are required to elucidate the molecular regulation for this possibility.

In conclusion, the present study has offered insight into the role of the IFITM3 gene in the pathogenesis of GC. An increased expression of IFITM3 was observed in GC tissues and cell lines, which is closely associated with the deregulation of Wnt signaling. Additionally, IFITM3 exhibits tumor promoter activity that facilitates the growth, migration, invasion and metastatic potential of tumor cells via the regulation of EMT and MMPs. These findings provide new and important information on the progression of GC and suggest that IFITM3 may be beneficial as a novel molecular target for the treatment of GC patients.

\section{Acknowledgements}

The present study was supported by the Department of Health of the Jiangsu Province Fund.

\section{References}

1. Bertuccio P, Chatenoud L, Levi F, et al: Recent patterns in gastric cancer: a global overview. Int J Cancer 125: 666-673, 2009.

2. Jemal A, Bray F, Center MM, Ferlay J, Ward E and Forman D: Global cancer statistics. CA Cancer J Clin 61: 69-90, 2011.

3. Cheng Y, Jin Z, Agarwal R, et al: LARP7 is a potential tumor suppressor gene in gastric cancer. Lab Invest 92: 1013-1019, 2012.

4. Khosravi Shahi P, Díaz Muñoz de la Espada VM, García Alfonso P, et al: Management of gastric adenocarcinoma. Clin Transl Oncol 9: 438-442, 2007.

5. Saitou M, Payer B, Lange UC, Erhardt S, Barton SC and Surani MA: Specification of germ cell fate in mice. Philos Trans R Soc Lond B Biol Sci 358: 1363-1370, 2003

6. Lange UC, Saitou M, Western PS, Barton SC and Surani MA: The fragilis interferon-inducible gene family of transmembrane proteins is associated with germ cell specification in mice. BMC Dev Biol 3: 1, 2003.

7. Lewin AR, Reid LE, McMahon M, Stark GR and Kerr IM: Molecular analysis of a human interferon-inducible gene family. Eur J Biochem 199: 417-423, 1991

8. Evans SS, Collea RP, Appenheimer MM and Gollnick SO: Interferon- $\alpha$ induces the expression of the L-selectin homing receptor in human B lymphoid cells. J Cell Biol 123: 1889-1898, 1993.

9. Tanaka SS, Yamaguchi YL, Tsoi B, Lickert H and Tam PP: IFITM/Mil/fragilis family proteins IFITM1 and IFITM3 play distinct roles in mouse primordial germ cell homing and repulsion. Dev Cell 9: 745-756, 2005.

10. Friedman RL, Manly SP, McMahon M, Kerr IM and Stark GR: Transcriptional and posttranscriptional regulation of interferoninduced gene expression in human cells. Cell 38: 745-755, 1984. 
11. Tanaka SS, Nagamatsu G, Tokitake Y, Kasa M, Tam PP and Matsui Y: Regulation of expression of mouse interferon-induced transmembrane protein like gene-3, Ifitm3 (mil-1, fragilis), in germ cells. Dev Dyn 230: 651-659, 2004.

12. Smith RA, Young J, Weis JJ and Weis JH: Expression of the mouse fragilis gene products in immune cells and association with receptor signaling complexes. Genes Immun 7: 113-121, 2006.

13. Saitou M, Barton SC and Surani MA: A molecular programme for the specification of germ cell fate in mice. Nature 418 293-300, 2002.

14. Nibbe RK, Markowitz S, Myeroff L, Ewing R and Chance MR: Discovery and scoring of protein interaction subnetworks discriminative of late stage human colon cancer. Mol Cell Proteomics 8: 827-845, 2009.

15. Seyfried NT, Huysentruyt LC, Atwood JA III, Xia Q, Seyfried TN and Orlando R: Up-regulation of NG2 proteoglycan and interferon-induced transmembrane proteins 1 and 3 in mouse astrocytoma: a membrane proteomics approach. Cancer Lett 263: 243-252, 2008.

16. Wylie C: IFITM1-mediated cell repulsion controls the initial steps of germ cell migration in the mouse. Dev Cell 9: 723-724, 2005.

17. Hatano H, Kudo Y, Ogawa I, et al: IFN-induced transmembrane protein 1 promotes invasion at early stage of head and neck cancer progression. Clin Cancer Res 14: 6097-6105, 2008.

18. Daniel-Carmi V, Makovitzki-Avraham E, Reuven EM, et al: The human $1-8 D$ gene (IFITM2) is a novel p53 independent proapoptotic gene. Int J Cancer 125: 2810-2819, 2009.

19. Li J, Yang B, Zhou Q, et al: Autophagy promotes hepatocellular carcinoma cell invasion through activation of epithelial-mesenchymal transition. Carcinogenesis 34: 1343-1351, 2013.

20. Ledford H: Cancer theory faces doubts. Nature 472: 273, 2011.

21. Gialeli C, Theocharis AD and Karamanos NK: Roles of matrix metalloproteinases in cancer progression and their pharmacological targeting. FEBS J 278: 16-27, 2011.

22. Roy R, Yang J and Moses MA: Matrix metalloproteinases as novel biomarkers and potential therapeutic targets in human cancer. J Clin Oncol 27: 5287-5297, 2009.

23. Andreu P, Colnot S, Godard C, et al: Identification of the IFITM family as a new molecular marker in human colorectal tumors. Cancer Res 66: 1949-1955, 2006.

24. Tian XH, Hou WJ, Fang Y, et al: XAV939, a tankyrase 1 inhibitor, promotes cell apoptosis in neuroblastoma cell lines by inhibiting Wnt/ $\beta$-catenin signaling pathway. J Exp Clin Cancer Res 32: 100 2013.
25. Zhao B, Wang $\mathrm{H}$, Zong $\mathrm{G}$ and Li P: The role of IFITM3 in the growth and migration of human glioma cells. BMC Neurol 13 210, 2013.

26. Li D, Peng Z, Tang H, et al: KLF4-mediated negative regulation of IFITM3 expression plays a critical role in colon cancer pathogenesis. Clin Cancer Res 17: 3558-3568, 2011.

27. Yang M, Gao H, Chen P, Jia J and Wu S: Knockdown of interferon-induced transmembrane protein 3 expression suppresses breast cancer cell growth and colony formation and affects the cell cycle. Oncol Rep 30: 171-178, 2013.

28. Kelly JM, Gilbert CS, Stark GR and Kerr IM: Differential regulation of interferon-induced mRNAs and c-myc mRNA by $\alpha$ - and $\gamma$-interferons. Eur J Biochem 153: 367-371, 1985.

29. Jiang D, Weidner JM, Qing M, et al: Identification of five interferon-induced cellular proteins that inhibit West Nile virus and dengue virus infections. J Virol 84: 8332-8341, 2010.

30. El-Tanani MK, Jin D, Campbell FC and Johnston PG: Interferoninduced transmembrane 3 binds osteopontin in vitro: expressed in vivo IFITM3 reduced OPN expression. Oncogene 29: 752-762, 2010.

31. Zhang YY, Chen B and Ding YQ: Metastasis-associated factors facilitating the progression of colorectal cancer. Asian Pac J Cancer Prev 13: 2437-2444, 2012.

32. Scheel C, Eaton EN, Li SH, et al: Paracrine and autocrine signals induce and maintain mesenchymal and stem cell states in the breast. Cell 145: 926-940, 2011.

33. Zhao Y, Guo Q, Chen J, Hu J, Wang S and Sun Y: Role of long non-coding RNA HULC in cell proliferation, apoptosis and tumor metastasis of gastric cancer: A clinical and in vitro investigation. Oncol Rep 31: 358-364, 2014.

34. Egeblad $\mathrm{M}$ and Werb Z: New functions for the matrix metalloproteinases in cancer progression. Nat Rev Cancer 2: 161-174, 2002.

35. Kabashima A, Maehara Y, Kakeji Y, Baba H, Koga T and Sugimachi K: Clinicopathological features and overexpression of matrix metalloproteinases in intramucosal gastric carcinoma with lymph node metastasis. Clin Cancer Res 6: 3581-3584, 2000.

36. Logan CY and Nusse R: The Wnt signaling pathway in development and disease. Annu Rev Cell Dev Biol 20: 781-810, 2004. 